\title{
BIOMIMETIC POLYMERS OF PLANT CUTIN: AN APPROACH FROM MOLECULAR MODELLING
}

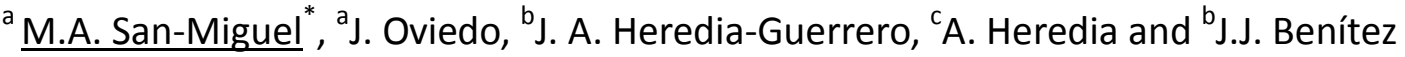 \\ ${ }^{a}$ Departamento de Química Física, Universidad de Sevilla, E-41012 Sevilla, Spain. \\ ${ }^{b}$ Instituto de Ciencia de Materiales de Sevilla (ICMS), CSIC-US, Consejo Superior de \\ Investigaciones Científicas (CSIC), Isla de la Cartuja, E-41092 Sevilla, Spain.

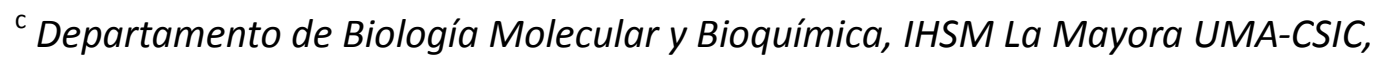 \\ Universidad de Málaga, E-29071 Málaga (Spain).
}

\footnotetext{
*e-mail: smiguel@us.es
}

\section{ABSTRACT}

Biomimetics of materials is based on adopting and reproducing a model in nature with a well-defined functionality optimized through evolution. An example is barrier polymers that protect living tissues from the environment. The protecting layer of fruits, leaves and non-lignified stems is the plant cuticle. The cuticle is a complex system in which the cutin is the main component. Cutin is a biopolyester made of polyhydroxylated carboxylic acids of 16 and 18 carbon atoms. The biosynthesis of cutin in plants is not well understood yet but a direct chemical route involving the self-assembly of either molecules or molecular aggregates has been proposed. In this work, we present a combined study using experimental and simulation techniques on self-assembled layers of monomers selectively functionalized with hydroxyl groups. Our results demonstrate that the number and position of the hydroxyl groups are critical for the interaction between single molecules and the further rearrangement. Also, the presence of lateral hydroxyl groups reinforces lateral interactions and favors the bi-dimensional growth (2D), while terminal hydroxyl groups facilitate the formation of a second layer caused by head-tail interactions. The balance of $2 \mathrm{D} / 3 \mathrm{D}$ growth is fundamental for the plant to create a protecting layer both large enough in 2D and thick enough in 3D. 


\section{KEYWORDS}

Cutin; Biopolyesters; AFM; MD simulations.

\section{INTRODUCTION}

In the past decades there has been a great effort to design environmental friendly polymeric materials replacing those obtained from petroleum. A successful example are biodegradable short-chain polyesters like polylactic acid and polyhydroxybutyrate, which, based on their hydrophobicity, are currently used as single-use packing material. However much less research has been focused on long-chain (16 carbon atoms and above) polyesters despite they are the base of ubiquitous plant barrier biopolymers like suberin and cutin. Understanding the formation and properties of these natural polymeric materials is an interesting route to design new non-toxic, fully biodegradable and water resistant products. Cutin, for instance, is a quite pure polyester present at the cuticle, the skin of fruits, seeds, leaves and green stems of higher plants. Chemically is composed by a mixture of $C_{16}$ and $C_{18}$ polyhydroxyacids being the 9(10), 16-dihydroxyhexadecanoic acid, by far, the most abundant [1]. Despite its early characterization in plant tissues, cutin biosynthesis is not yet well understood. Enzymatic and gene transcription routes are still under discussion. However, a direct chemical synthesis from either self-assembled molecules or aggregates has been proposed recently [2]. Thus, the understanding of the intermolecular interaction between monomeric units is a key issue. To address this point, we propose model systems in which the aliphatic skeleton of the molecule is selectively functionalized with hydroxyl groups in primary and secondary positions and placed on top of an inert flat support in the form of self-assembled layers. In particular, we have selected palmitic acic (PA), 16hydroxyhexadecanoic acid (HPA) and the 9,16-dihydroxypalmitic acid (diHPA) as reference monomers without $-\mathrm{OH}$ groups, with a terminal hydroxyl group and with a terminal and a secondary $-\mathrm{OH}$ groups, respectively.

Results obtained from molecular dynamics (MD) simulations are compared with experimental Atomic Force Microscopy (AFM) data to determine the role of the specific hydroxyl functionalization in the structure of the self-assembled layer. This information will be used to design the right monomeric composition leading to consistent polymeric films. 


\section{METHODS}

Molecular dynamics (MD) simulations were carried out using DL_POLY code [3-4] in the canonical ensemble (NVT). The initial velocities were set up from a Boltzmann distribution. The equations of motion were integrated by using the velocity-Verlet algorithm with a time step of $1 \mathrm{fs}$. The temperature was kept constant at $300 \mathrm{~K}$ by applying a NoséHoover thermostat [5-6].

The initial configurations for the monolayer systems were built by placing 100 molecules (all-trans configuration) with the $-\mathrm{COOH}$ group facing the surface and arranged in a triangular lattice with a distance of $5.2 \AA$ to the nearest neighbors yielding a surface density $\left(\rho_{s}\right)$ of $21.4 \AA^{2} /$ molecule. Periodic conditions were imposed along the $X$ and $Y$ directions in a box of dimensions of $L_{x}=52.0 \AA, L_{y}=45.0 \AA$ and $L_{z}=200.0 \AA$.

A cutoff radius of $15 \AA$ was used for the Lennard-Jones (6-12) potential terms. The electrostatic interactions were treated using the method of Hautman and Klein which is an adaptation of the Ewald method for systems which are periodic in two dimensions only. [7]

Simulations were extended up to $10 \mathrm{~ns}$ for the monolayer systems and $4 \mathrm{~ns}$ for the bilayer systems to assure equilibrium conditions. Configurations were saved every 1000 steps (1 ps) and the statistical analysis was done for the last nanosecond.

The interatomic potential parameters have been used already in previous studies of similar systems [8-10]. Alkane chains were modelled with a united atom potential, based on CHARMM22 but with parameters adjusted to give a better description of the crystal structure of n-alkanes [11]. The other atoms in the hydroxyl and carboxylic groups were represented explicitly [12-13].

The substrate was characterized as a flat surface, and the interaction between each particle and the surface was computed according to

$$
V(r)=\frac{C_{12}}{\left(z-z_{0}\right)^{12}}-\frac{C_{3}}{\left(z-z_{0}\right)^{3}}
$$

where $C_{n} \propto\left(\sigma \cdot \varepsilon^{n}\right)^{1 / 2}, z$ is the distance to the surface, and $z_{0}$ is a limit approach distance for each center. The parameters $\varepsilon$ and $\sigma$ are Lennard-Jones potential parameters, and they are estimated by means of the Lorentz-Berthelot combining rules. $\mathrm{C}_{n}$ parameters for $\mathrm{CH}_{2}$ and $\mathrm{O}$ were taken from the literature [14-15]. 


\section{RESULTS}

\section{Monolayer structures}

We have chosen a series of molecules containing hydroxyl groups in different positions along the alkyl chain to study the self-assembly process on muscovite mica surface as a reference inert flat support. The palmitic acid (PA) has been selected as a reference molecule with no hydroxyl groups, the 16-hydroxyhexadecanoic acid (HPA) with a terminal hydroxyl group (primary -OH group) and the 9,16-dihydroxypalmitic acid (diHPA) which has both a primary and a secondary $-\mathrm{OH}$ groups.

Topographic Atomic Force Microscopy images indicate that PA do self-assemble on mica in the form of dendritic monolayer islands typically $700-800 \mathrm{~nm}$ in diameter and about 1.3-1.5 nm high (see Figure 1a). This island height corresponds with a tilted arrangement of molecules exposing their hydrophobic methylene end, as inferred from friction and adhesion AFM mapping. Therefore, the PA molecules interact with mica surface through the carboxylic group, likely by proton exchange with $\mathrm{K}^{+}$ions from mica and the formation of the corresponding carboxylate. Obtaining low saturation coverage (10-15\%) despite the palmitic acid solution concentration used supports this later argument.

Hydrophilic $-\mathrm{COOH}$ groups bond to the surface but, simultaneously, they may interact with neighbor $-\mathrm{COOH}$ groups to form a hydrogen bonding network. Molecular dynamics simulations have been conducted to gain insight into the role of such hydrogen bonding on the self-assembly process of palmitic acid on mica.

Results from MD simulations indicate a clear tendency to form well self-assembled aggregates (or islands in a higher order of size magnitude which is out of our simulation scale) together with an unstructured group of palmitic acid molecules connected by a hydrogen bonding network (see Figure 2a). Such unstructured phase is considered to be responsible for the low coverage, dendritic and low height island observed by AFM.

Next, we consider the modifications induced by the presence of a primary (terminal) hydroxyl group in the 16-hydroxyhexadecanoic acid. Addition of an end hydroxyl group to the alkyl chain drastically changes the growing pattern when compared with palmitic acid. Molecular self-assembly of 16-hydroxyhexadecanoic acid forms elongated and terraced multilayered islands with steps heights of $2.5 \mathrm{~nm}$, which is quite similar to the all-trans 
configuration length $(2.2 \mathrm{~nm})$, Figure $1 \mathrm{~b}$.

MD simulations of a 16-hydroxyhexadecanoic acid monolayer were carried out using a similar protocol than for palmitic acid. The structure of an equilibrated 16hydroxyhexadecanoic acid is shown in Figure $2 \mathrm{~b}$. Incorporation of a terminal hydroxyl group in the molecule causes the formation of a second hydrogen bond network which significantly reduces the molecular tilt increasing so the layer thickness in agreement with the AFM observations. This evidence can be monitored by inspecting the angle formed between the tail vector and the normal surface. The tail vector has been defined as the eigenvector associated to the smallest eigenvalue of the moment of inertia tensor for all carbon atoms in the alkane chain. This corresponds to that principal axis about which rotation is the easiest. We have computed the tilt angle distribution for both palmitic and 16-hydroxyhexadecanoic acid monolayers and results are plotted in Figure 3. Palmitic acid shows a bi-modal distribution. One with maximal intensity at $30^{\circ}$ corresponds to those molecules in compact aggregates, and a second one at high tilt angles (close to $90^{\circ}$ ) arising from those unassembled molecules. In contrast, the tilt distribution for 16-hydroxyhexadecanoic acid is ranged between 20 and $40^{\circ}$ as a consequence of a more effective self-assembly process.

The third molecule studied (9,16-dihydroxypalmitic acid, diHPA) is a fatty acid that contains both a primary (end) and a secondary (mid-chain) hydroxyl groups. This molecule is the main monomer extracted after plant cutin depolymerization. The results from MD simulation of a monolayer indicate the formation of two hydrogen bonding networks: one resulted from interactions between secondary hydroxyl groups and another between primary $-\mathrm{OH}$ groups. Thus, the monolayer exhibits a tilt angle distribution which, differently from the 16-hydroxydecanoic acid, is peaked at $35^{\circ}$ as consequence of a more compact structure. This is in remarkable agreement with the AFM observations indicating that this molecule forms interconnected and uniform islands with a high surface coverage, Figure 1c.

Compared with other hydroxylated fatty acids, the first monolayer of diHPA develops much faster and in a larger extent than the one corresponding to palmitic acid, in which no secondary hydroxyl groups favor 2D growth. Besides, the monolayer formation stage is welldefined in diHPA while it is not observable in HPA, which readily goes to a 3D growing pattern. The effective formation of the monolayer in diHPA can be explained by a 
cooperation mechanism involving both secondary and primary hydroxyl groups. The network of hydrogen bonding between primary - $\mathrm{OH}$ groups is reinforced by a second network created by bridging secondary hydroxyl groups (Figure 2c).

\section{Formation of multilayer systems}

However, the most important feature of the self-assembly process of 16hydroxyhexadecanoic acid on mica is the formation of multilayered islands. Assuming that 16-hydroxyhexadecanoic acid bonds to mica through the carboxyl end, the presence of exposed hydroxyl groups causes competition between adsorption on mica to create new islands (2D growth) and adsorption on top of an existing monolayer (3D growth). In the second case, the exposed terminal hydroxyl groups trigger multilayer growth and additional head to tail (carboxyl to hydroxyl groups) hydrogen bonds are created.

Topographic AFM measurements do not provide information about the molecular orientation in the self-assembled monolayers of 16-hydroxyhexadecanoic acid, i.e. whether the molecule bonds to the surface through the $-\mathrm{COOH}$ or $-\mathrm{OH}$ group. Previous AFM results show that fatty acid SAMs on mica are more stable than those corresponding to the analogous primary alcohol [16]. This fact allows us to assume reasonably that the adsorption occurs preferentially by the carboxylic group. Furthermore, previous STM studies on selfassembly of fatty hydroxyl acids on HPOG have shown that $-\mathrm{COOH} \bullet \bullet \bullet \mathrm{HOOC}$ - interaction is stronger than $-\mathrm{COOH} \bullet \bullet \mathrm{HO}-[17-18]$. This observation may cause competition between head to head (-COOH$\bullet \bullet \bullet \mathrm{HOOC}-$ ) and head to mica bonding and reverse orientation (bonded to mica through the hydroxyl group) of a fraction of molecules in the self-assembled system. As first approximation in our simulations, we have assumed that molecules in the monolayer adsorb to the surface only through the $-\mathrm{COOH}$. Then, the molecules in the second layer interact with the exposed $-\mathrm{OH}$ groups only by the $-\mathrm{COOH}$ group. Thus, to model a bilayer of both palmitic and 16-hydroxyhexadecanoic acids, a second equilibrated layer was placed at 4 $\AA$ from the highest atoms in the monolayer previously equilibrated. Then, simulations were extended for 4 ns. Figure 4 shows a side view of the final configuration for both systems. It is clear that the palmitic acid bilayer is hindered because interaction between the hydrophobic tail in the first layer and the hydrophilic group in the second layer is not effective. A very 
different scenario is observed for 16-hydroxyhexadecanoic acid, where terminal hydroxyl groups in the first layer interact with carboxylic groups in the second layer by hydrogen bonding, leading to a well-organized self-assembled bilayer.

When both primary and secondary hydroxyl groups are present, the self-assembly process is a combination of the two patterns already described. Secondary hydroxyl groups favour 2D growth of islands while primary hydroxyls promote formation of multilayers. This is clearly seen in Figure 1c, where evolution of 9,16-dihydroxypalmitic acid (diHPA) layers vs. preparation solution concentration is shown. At low concentration the first monolayer develops with a minor but growing presence of the second monolayer as the concentration is raised. Full coverage of mica support is achieved above $2 \mathrm{mM}$. The system further develops until the first monolayer is also fully covered by a second one and, finally, at the highest concentration, a three layers deposit predominates before large particles precipitation occurs.

Results from the MD simulations of a diHPA bilayer indicate also the formation of a well-developed system in which the interactions between the secondary hydroxyl groups influence significantly on the molecular orientation. It can be seen from Figure 4, that despite the diHPA molecules do not show all-trans conformations as HPA, they still selfassemble in a compact structure.

Therefore, for HPA the multilayer system is developed from interactions between the terminal $-\mathrm{OH}$ groups of a formed layer and the $-\mathrm{COOH}$ groups of new molecules approaching. Thus, the emerging hydrogen bonding networks are flexible enough to allow the alkane chains to keep the most favorable conformation (all-trans) and the effective packing between them leads to a tilt angle distribution peaked at $38^{\circ}$ (Figure 5). On the other hand, the secondary $-\mathrm{OH}$ group of the diHPA molecules introduces additional hydrogen bonding network dominating the lateral molecular interactions and leading to a selfassembled system with a molecular orientation close to the verticality (tilt angle distribution c.a. $\left.10-20^{\circ}\right)$.

\section{CONCLUSIONS}

We have reported a combined study of experimental and computer simulation 
techniques on self-assembled systems of three different monomers selectively functionalized with hydroxyl groups on mica surface acting as an inert support. Our results show that palmitic acid (PA) forms well self-assembled islands together with unstructured aggregates which are responsible for the low coverage observed in the AFM images. The presence of a terminal hydroxyl group in 16-hydroxyhexadecanoic acid (HPA) allows more efficient lateral molecular interactions achieving high monolayer coverages and also triggers the formation of multilayer structures (3D growth). The addition of a secondary hydroxyl group in the 9,16dihydroxypalmitic acid (diHPA) allows for another hydrogen bonding network improving the bi-dimensional growth (2D) and thus the first monolayer develops much rapidly. Furthermore, the multilayer growth also occurs but always accompanied by a high coverage of the last layers before additional layers start to develop. Therefore, to create an effective protecting layer in the plant, the self-assembly process has to balance both growths, being large enough in 2D and thick enough in 3D. The first one is mainly controlled by interactions between secondary hydroxyl groups and the second one by primary hydroxyl and acid groups.

\section{ACKNOWLEDGEMENTS}

Funding is provided by the Spanish Ministerio de Economía y Competitividad under project CTQ2011-24299 and by Junta de Andalucía grant TEP-7418 under the Motriz Program.

\section{REFERENCES}

[1] Arrieta-Baez D, Cruz-Carrillo M, Gómez-Patiño MB, Zepeda-Vallejo LG (2011) Derivatives of 10,16-dihydroxyhexadecanoic acid isolated from tomato (Solanum lycopersicum) as potential material for aliphatic polyesters. Molecules 16: 4923-4936. doi: 10.3390/molecules16064923

[2] Domínguez E, Heredia-Guerrero JA, Benítez JJ, Heredia A (2010) Self-assembly of supramolecular lipid nanoparticles in the formation of plant biopolyester cutin. Mol BioSyst 6: 948-950. doi: 10.1039/B927186D

[3] Smith W, Yong CW, Rodger PM (2002) DL_POLY: application to molecular simulation. Mol Simul 28: 385-471. doi: 10.1080/08927020290018769 
[4] Available at www.ccp5.ac.uk/DL POLY

[5] Nose S (1984) A unified formulation of the constant temperature molecular dynamics methods. J Chem Phys 81: 511-519.

[6] Nose S (2002) A molecular dynamics method for simulations in the canonical ensemble. Mol Phys 100: 191-198. 10.1080/00268970110089108

[7] Hautman J, Klein ML (1992) Mol Phys 75: 379-395. doi:10.1080/00268979200100301

[8] Heredia-Guerrero JA, San-Miguel MA, Sansom MSP, Heredia A, Benitez JJ (2009) Chemical Reactions in 2D: Self-Assembly and Self-Esterification of 9(10),16-Dihydroxypalmitic Acid on Mica Surface. Langmuir 25: 6869-6874. doi: 10.1021/la9001412

[9] Heredia-Guerrero JA, San-Miguel MA, Sansom MSP, Heredia A, Benitez JJ (2010) Aleuritic (9,10,16-trihydroxypalmitic) acid self-assembly on mica. Phys Chem Chem Phys 12: 1042310428. doi: 10.1039/c0cp00163e

[10] Heredia-Guerrero JA, San-Miguel MA, Luna M, Domínguez E, Heredia A, Benítez JJ (2011) Structure and support induced structure disruption of soft nanoparticles obtained from hydroxylated fatty acids. Soft Matter 7: 4357-4363. doi: 10.1039/c0sm01545h

[11] San-Miguel MA, Rodger PM (2001) Simulation of Deposition of Wax to Iron Oxide Surfaces. Mol Simul 26: 193-216. doi: 10.1080/08927020108028293

[12] Jorgensen WL (1986) Optimized Intermolecular Potential Functions for Liquid Alcohols. J Phys Chem 90: 1276-1284.

[13] Briggs JM, Nguyen TB, Jorgensen WL (1991) Monte Carlo Slmulations of Liquid Acetic Acld and Methyl Acetate with the OPLS Potential Functions. J Phys Chem 95: 3315-3322.

[14] Hautman J, Klein ML (1989) Simulation of a monolayer of alkyl thiol chains. J Chem Phys 91: 4994-5001.

[15] Fartaria RPS, Freitas FFM, Silva Fernandes FMS (2005) A study of 1-decanethiol selfassembly on gold electrodes by computer simulation. J Electroanal Chem 574: 321-331. doi:10.1016/j.jelechem.2004.08.015

[16] Benítez JJ, Heredia-Guerrero JA, Heredia A (2007) Self-assembly of carboxylic acids and hydroxyl derivatives on mica. A qualitative AFM study. J Phys Chem C 111: 9465-9470. doi: 10.1021/jp070563y

[17] Cyr DM, Venkataraman B, Flynn GW (1996) STM investigations of organic molecules 
physisorbed at the liquid-solid interface. Chem Mater 8: 1600-1615.

[18] Oian P, Nanjo H, Yokoyama T, Suzuki TM (1999) STM observation of 12hydroxyoctadecanoic acid and its 4,4'-bipyridinium salt self-assembled on a graphite surface. Chem Commun 13: 1197-1198. 


\section{FIGURE CAPTIONS}

Figure 1. Topographic AFM images. A) Palmitic acid forms dendritic monolayer islands. B) HPA forms elongated and terraced multilayered islands with steps heights $(2.5 \mathrm{~nm})$ very similar to the extended molecule length $(2.2 \mathrm{~nm})$. C) The growth of diHPA multilayers on mica from solutions with increasing concentration (mM).

Figure 2. Side views of monolayers systems after 10 ns MD simulations. a) Palmitic acid (PA); b) 16-hydroxyhexadecanoic acid (HPA); c) 9,16-dihydroxypalmitic acid (diHPA). Oxygen and hydrogen atoms in $-\mathrm{OH}$ and $-\mathrm{COOH}$ groups are represented by red and white spheres respectively.

Figure 3. Tilt angle distributions for monolayers of PA, HPA and diHPA.

Figure 4. Side views of bilayers systems after 4 ns MD simulations. a) Palmitic acid (PA); b) 16-hydroxyhexadecanoic acid (HPA); c) 9,16-dihydroxypalmitic acid (diHPA). Oxygen and hydrogen atoms in $-\mathrm{OH}$ and $-\mathrm{COOH}$ groups are represented by red and white spheres respectively.

Figure 5. Tilt angle distributions for bilayers of HPA and diHPA. 
(a)
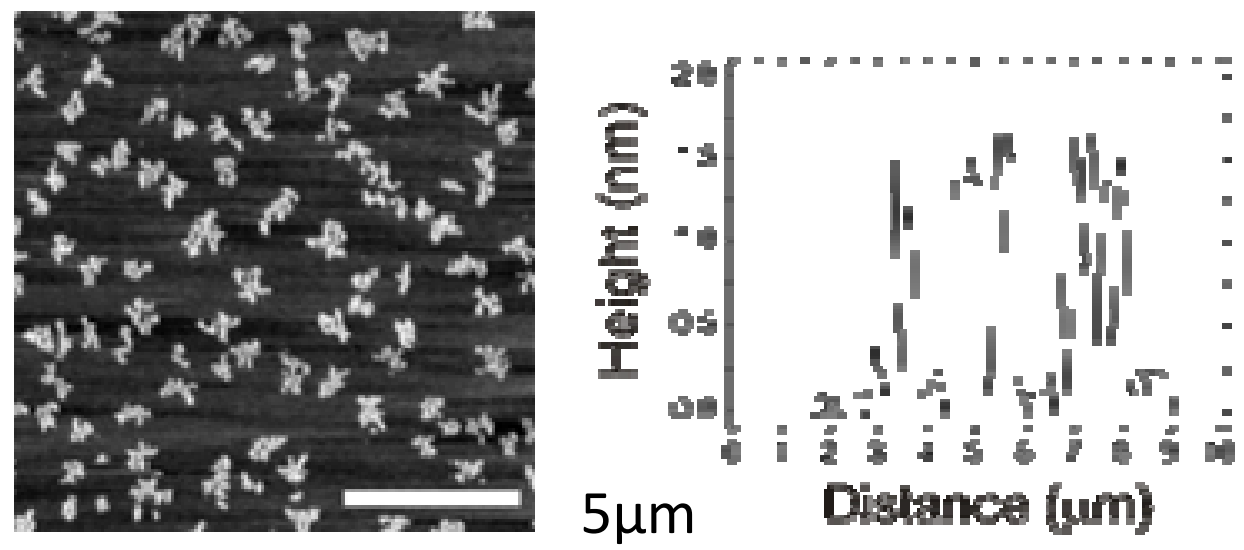

(b)
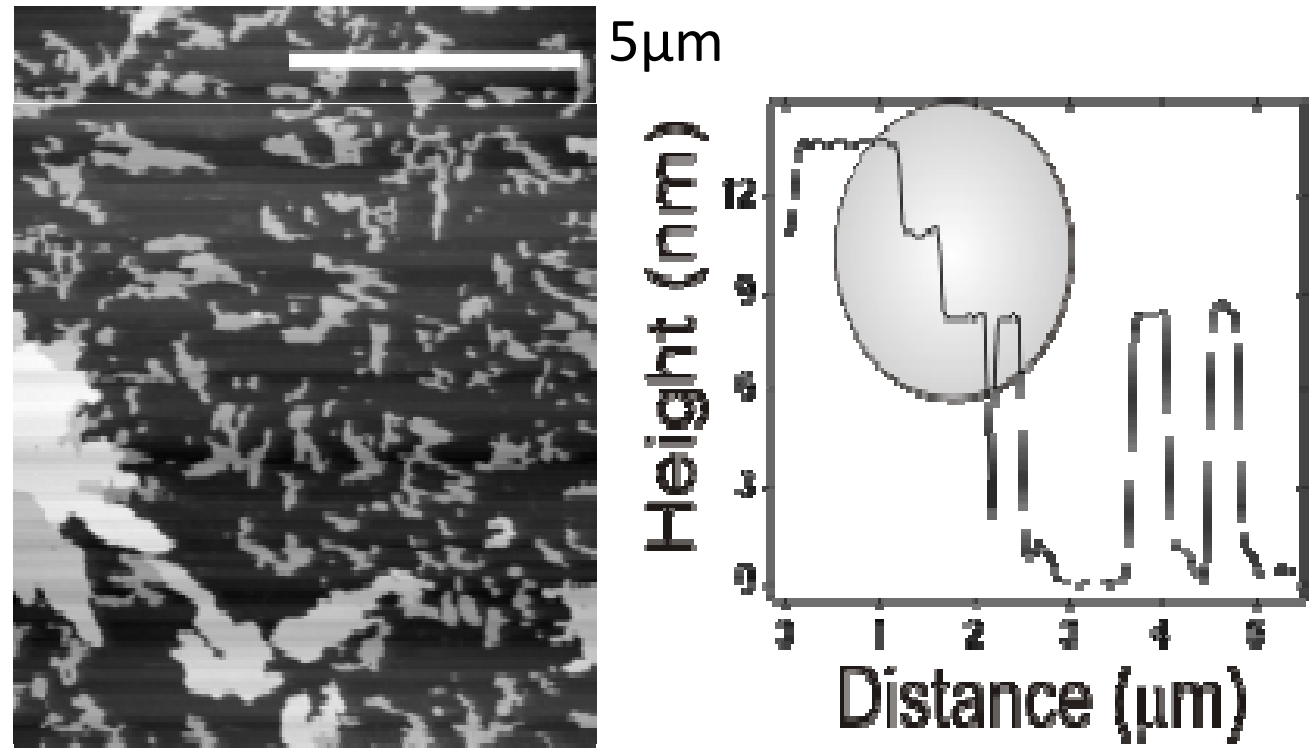

(c)
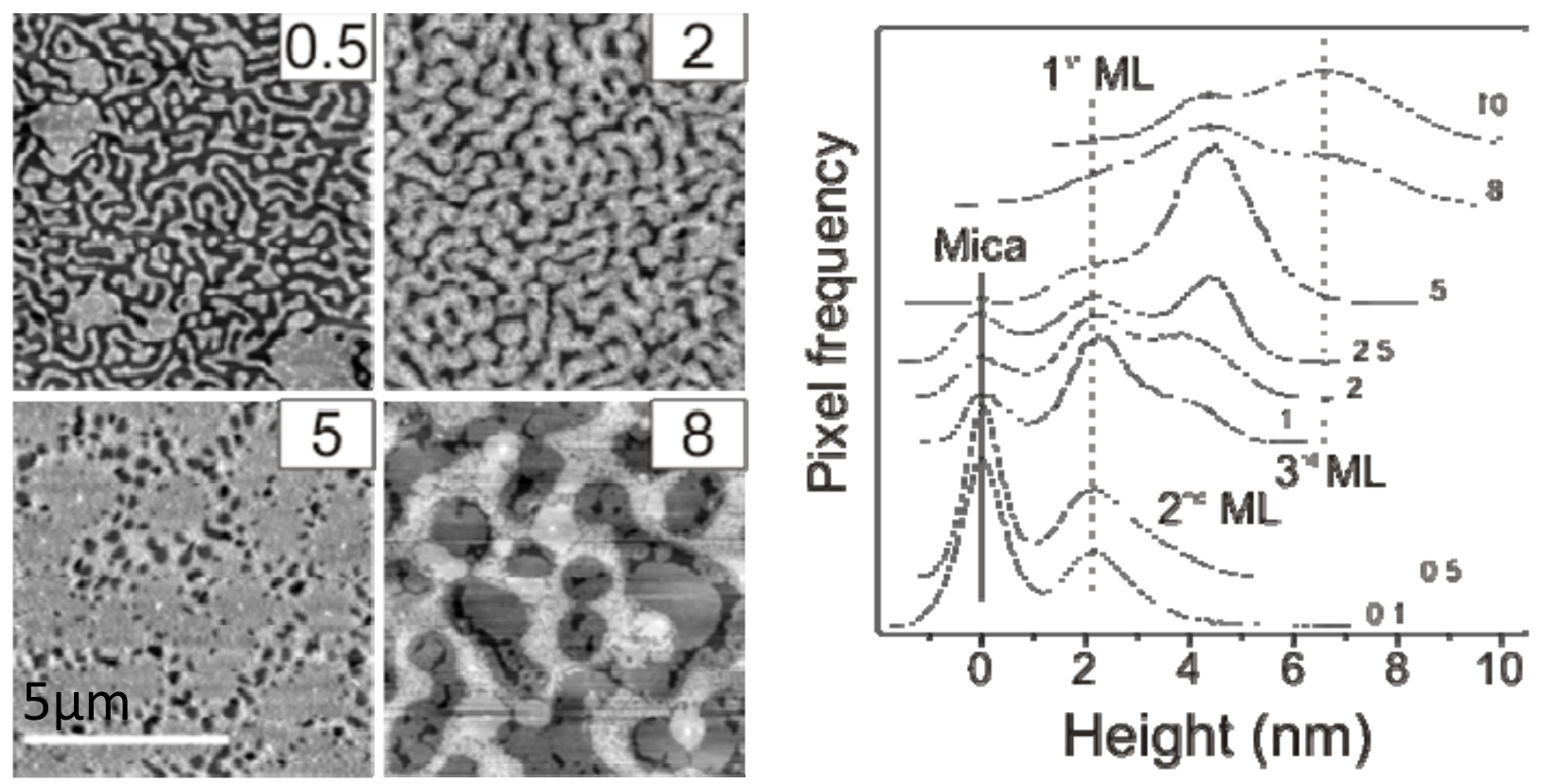

Figure 1 
(a)

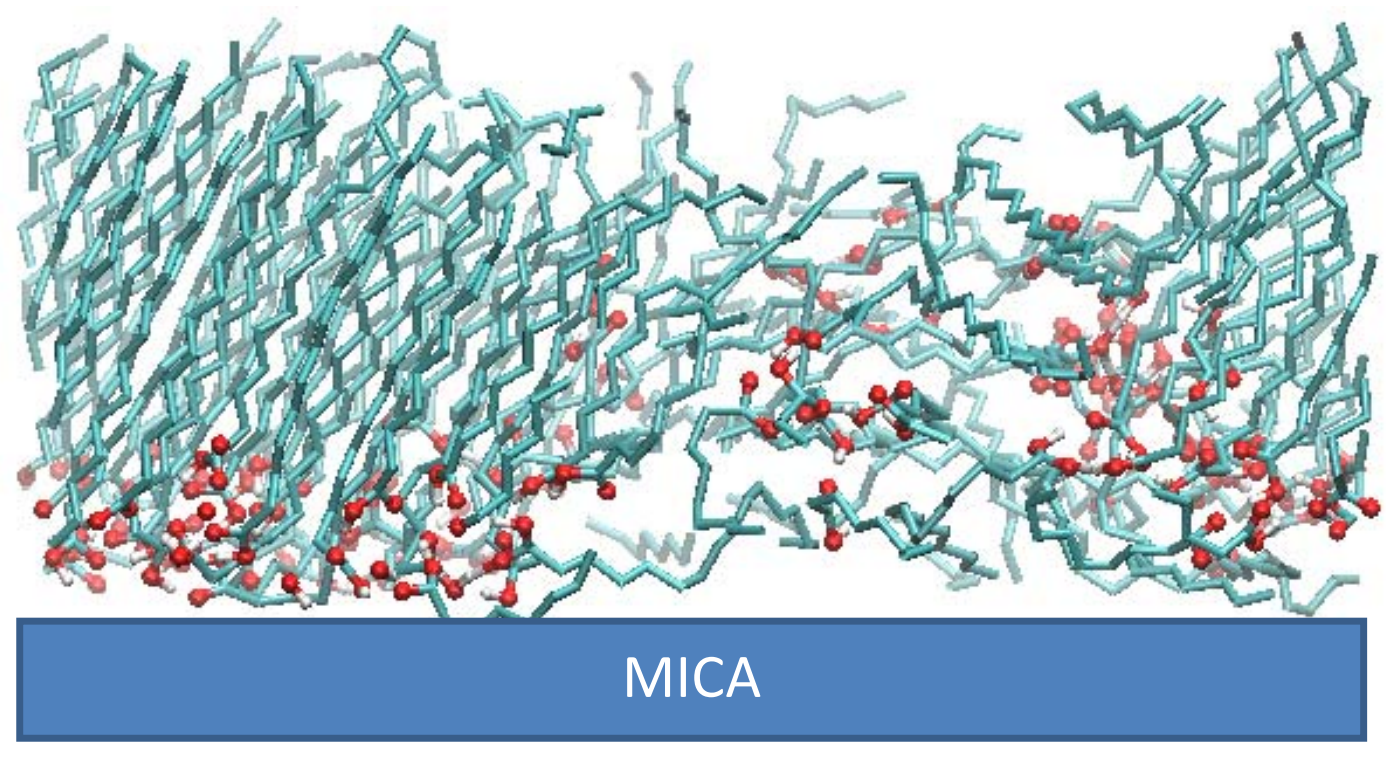

(b)

(c)
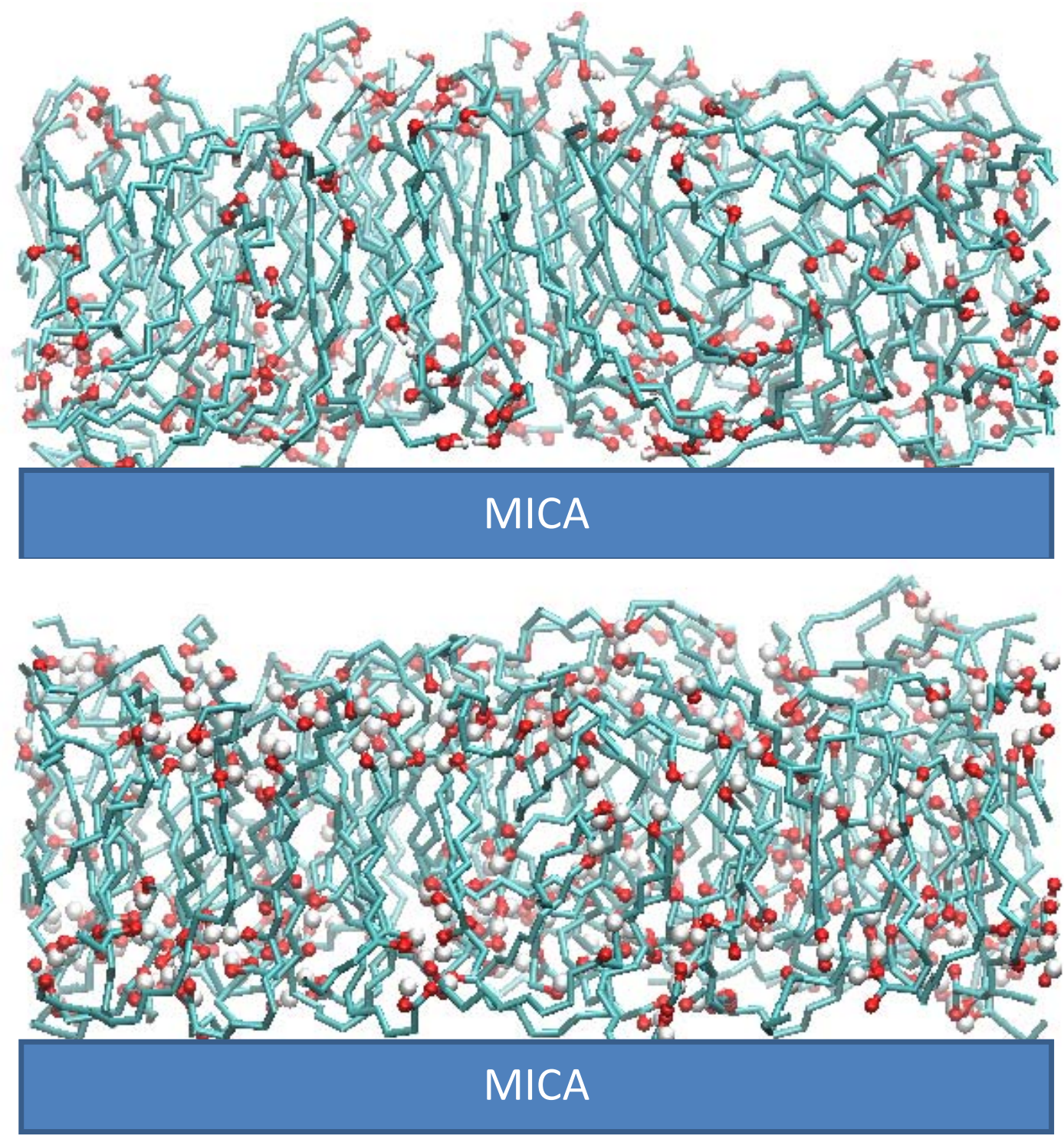

Figure 2 


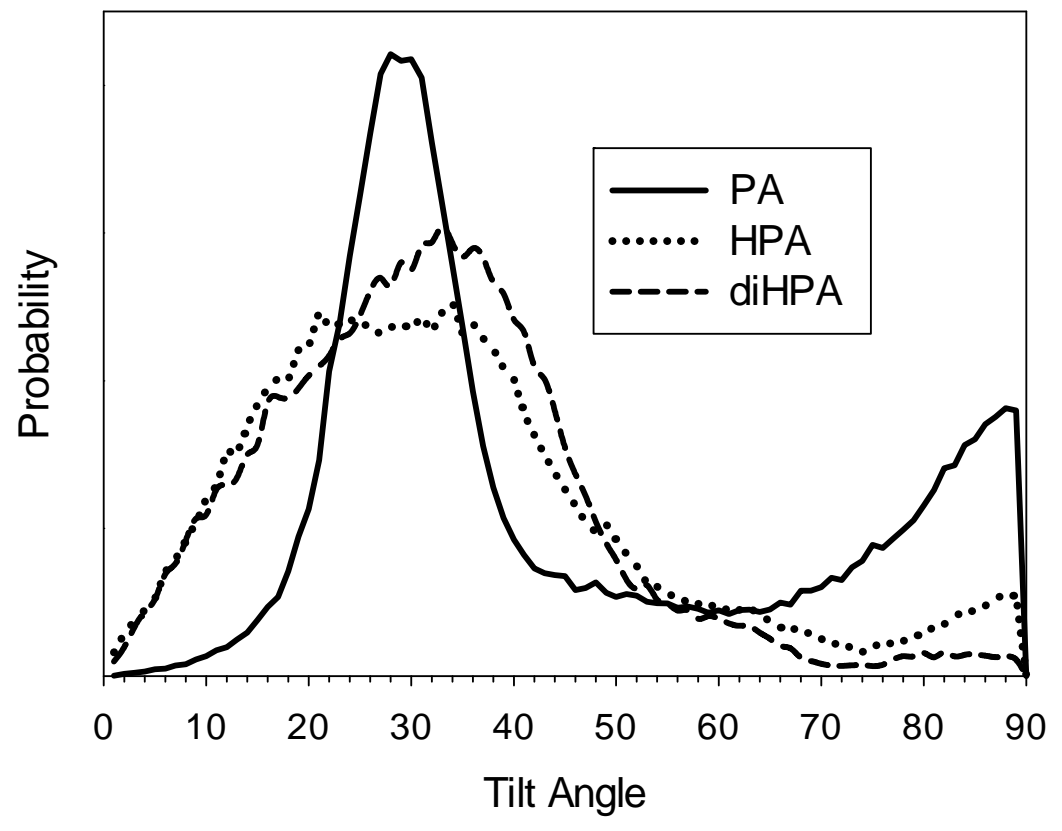

Figure 3 
(a)

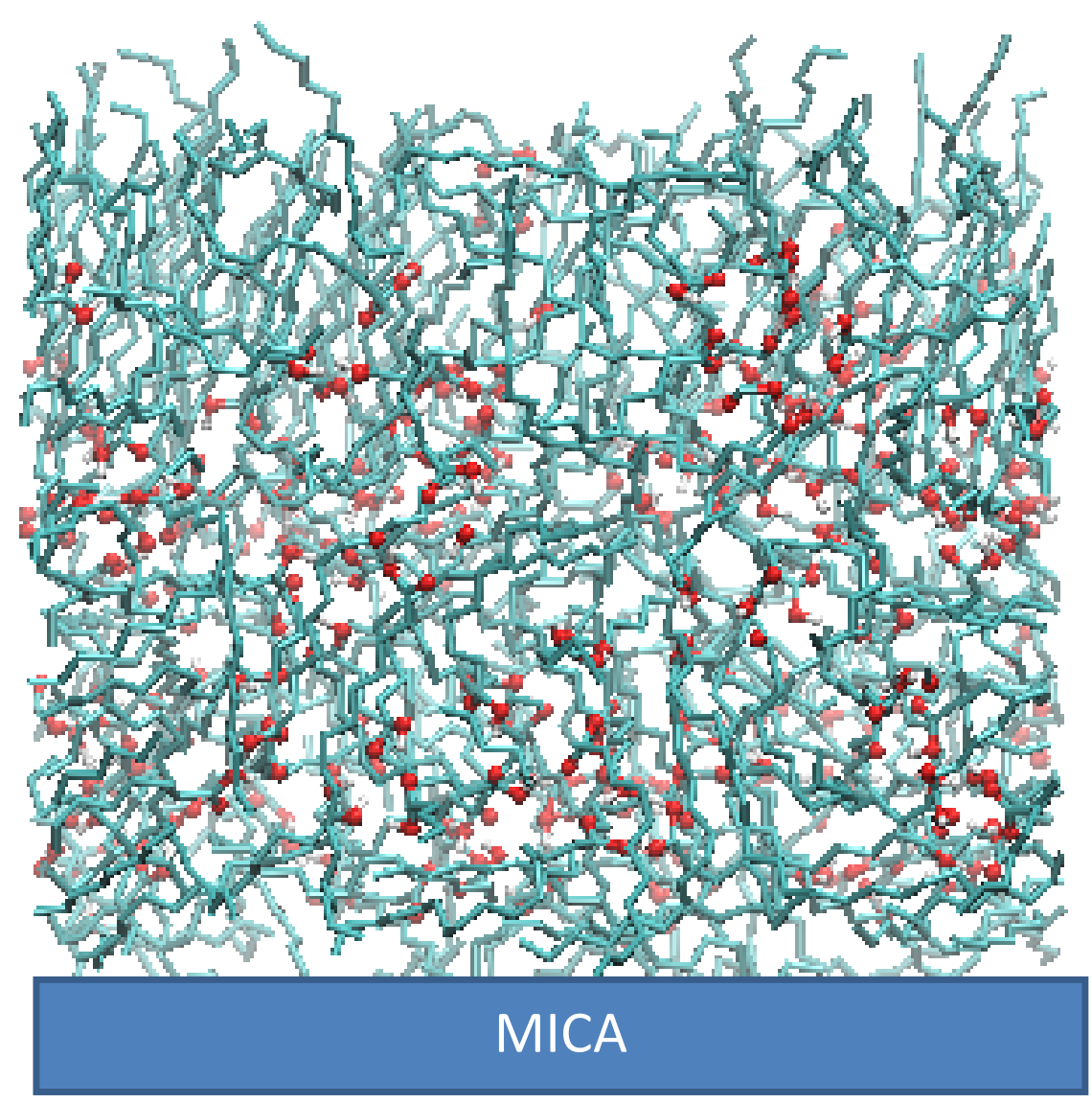

(b)

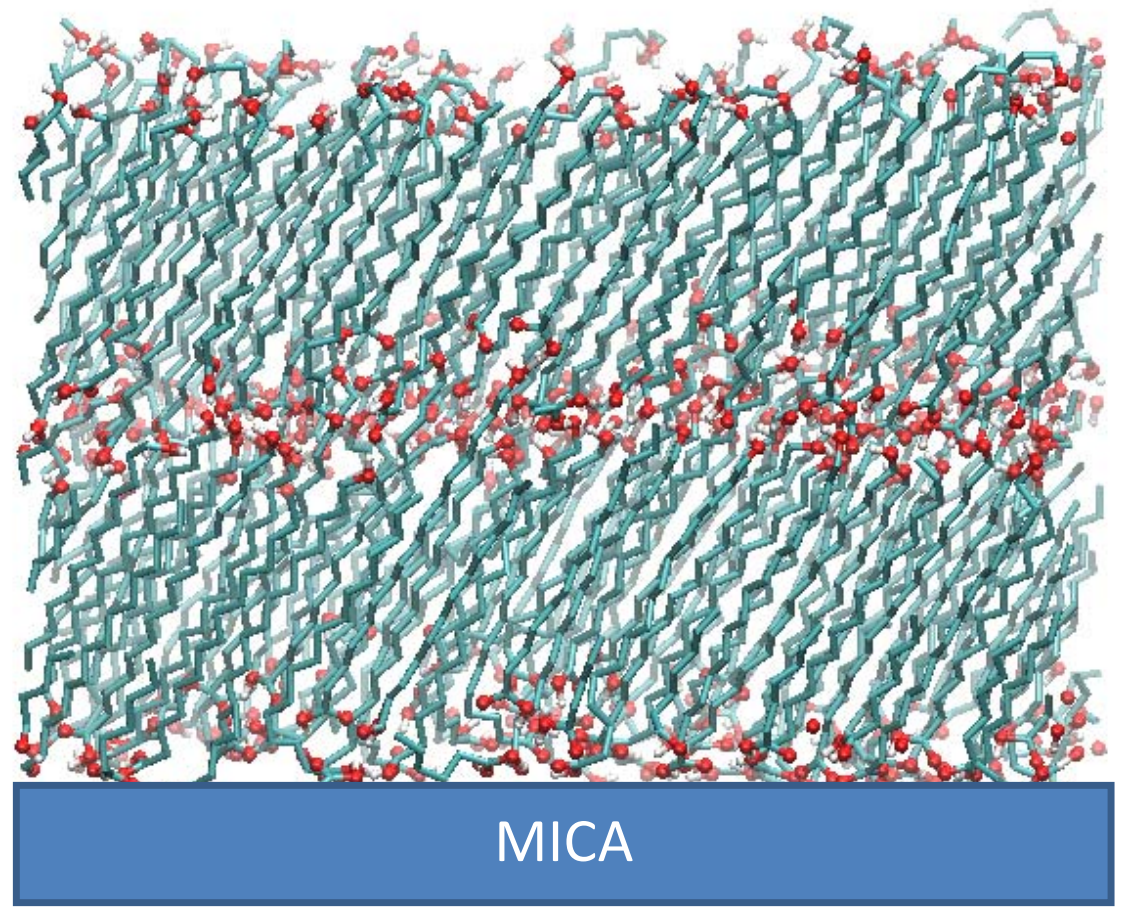


(c)

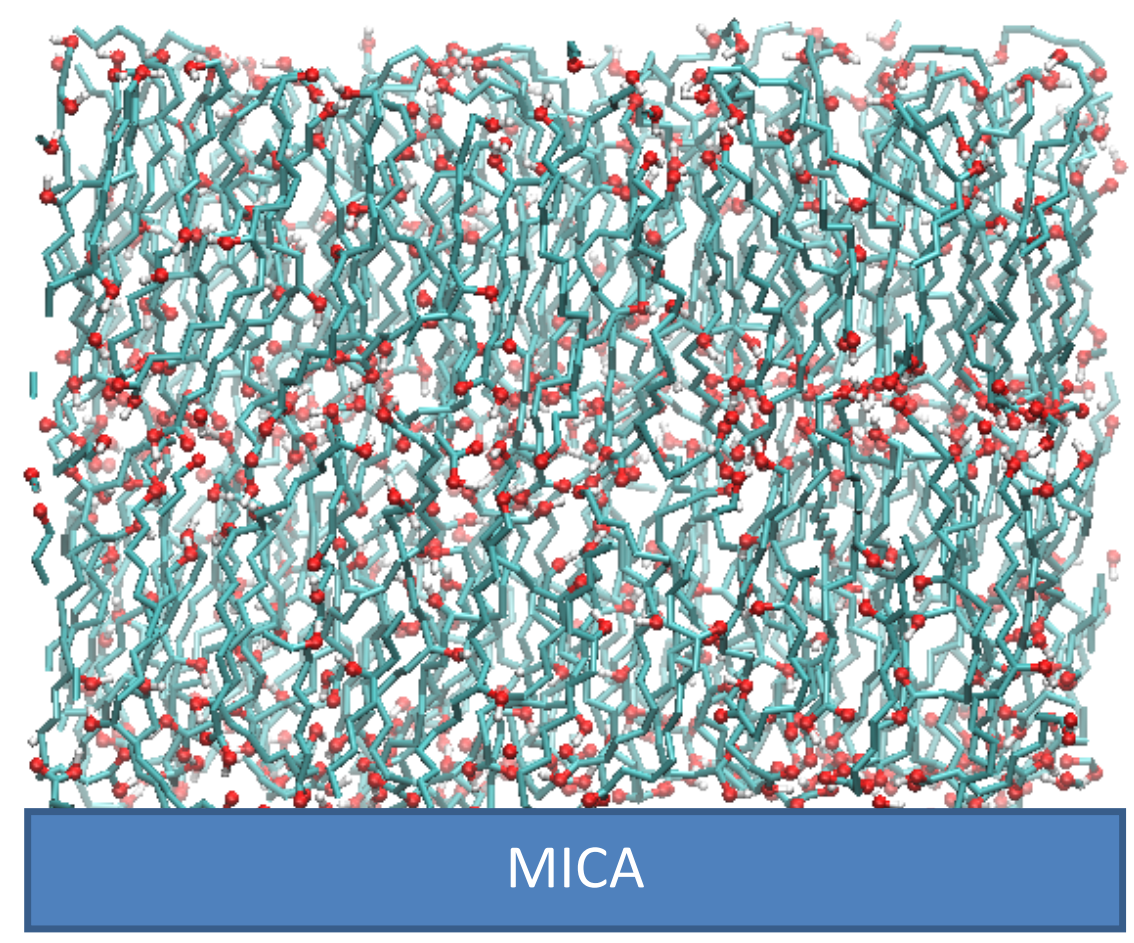

Figure 4 


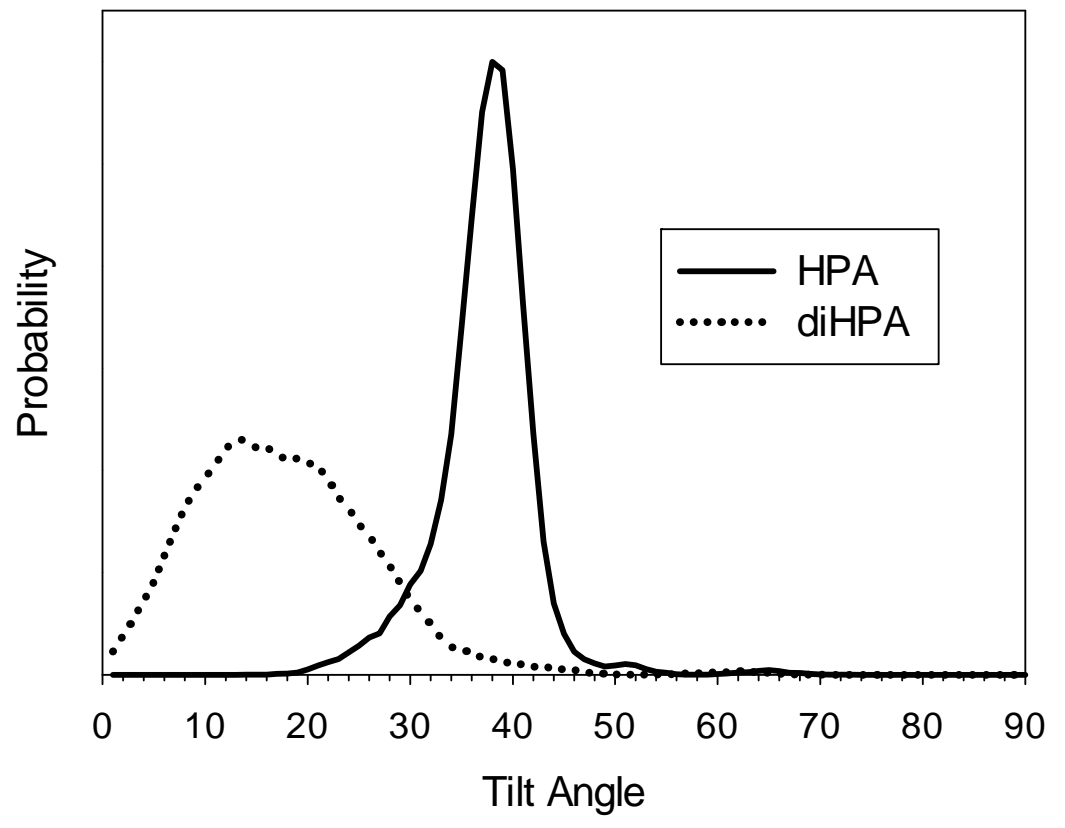

Figure 5 